

\section{Sumário}

I. Crônicas do Direito Internacional ...............................................1

REPARAÇÃo DE VÍTIMAS À LUZ DE UM TRATAdo SOBRE EMPRESAS E DIREITOS HUMANOS ............ 3 Ana Cláudia Ruy Cardia

Consumer Social Responsibility as a Requirement for Corporate Social ResponsibiliTY

Nitish Monebhurrun

Crisis in Venezuela: The Brazilian response to the massive flow of Venezuelans in

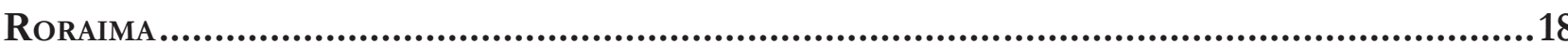

Jacqueline Salmen Raffoul

II. Dossiê EsPecial: Business and Human Rights.........................................23

Some remarks on the third sessions of the Business and Human Rights Treaty Pro-

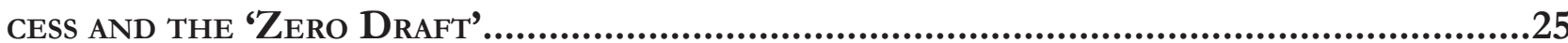

Humberto Cantú Rivera

The United Nations guiding principles on business and human Rights, the State

DUTY TO PROTECT HUMAN RIGHTS AND THE STATE-BUSINESS NEXUS.

Mihaela Maria Barnes

HARdening SOFT LAW: ARE THE EMERging Corporate SOCIAL DisClOSURE LAWS CAPABLE OF GENERATING SUBSTANTIVE COMPLIANCE WITH HUMAN RIGHTS?

Justine Nolan

Del Documento de Elementos al Draft 0: apuntes jurídicos respecto del posible CONTENIDO DEL PROYECTO DE INSTRUMENTO VINCULANTE SOBRE EMPRESAS TRANSNACIONALES

Y OTRAS EMPRESAS CON RESPECTO A LOS DERECHOS HUMANOS

Adoración Guamán 
ACCESS TO REMEDIES AND THE EMERGING ETHICAL DILEMMAS: CHANGING CONTOURS WITHIN THE BUSINESS-HUMAN RIGHTS DEBATE

Justin Jos

LA RESPONSABILIDAD PENAL DE LAS EMPRESAS POR GRAVES VIOLACIONES DE DERECHOS HUMANOS: PRÁCTICA ACTUAL Y DESAFÍOS FUTUROS 130

Daniel Iglesias Márquez

THE ENVIRONMENTAL LAW DIMENSIONS OF AN INTERNATIONAL BINDING TREATY ON BUSINESS AND HUMAN RIGHTS

Juan Gabriel Auz Vaca

Los Objetivos de Desarrollo Sostenible en Europa y su Intersección con el Marco de los Negocios y los Derechos Humanos

Paolo Davide Farah

HumAN RIGHTS AND MARKET ACCESS 203

Danielle Mendes Thame Denny

BusinesS AND HUMAN RIGHTS IN BRAZIL: EXPLORING HUMAN RIGHTS DUE DILIGENCE AND OPERATIONAL-LEVEL GRIEVANCE MECHANiSMS IN THE CASE OF KinRoss PARACATU Gold Mine...222 Mariana Aparecida Vilmondes Türke

Human Rights and eXtractive industries in Latin America: What Responsibility of CORPORATIONS AND THEIR STATES OF ORIGIN FOR HUMAN RIGHTS VIOLATIONS IN THE INTER-American Human Rights System?.

Alberto do Amaral Junior e Viviana Palacio Revello

MultinaCiOnAIS FAST FASHION E DIREITOS HUMANOS: EM BUSCA DE NOVOS PADRÕES DE RESPONSABILIZAÇÃO 255

Laura Germano Matos e João Luis Nogueira Matias

III. Artigos sobre outros temas

EFFICIENCY AND EFFICACY OF PUBLIC FOOD PROCUREMENT FROM FAMILY FARMERS FOR SCHOOL FEEDING IN BRAZIL. 271

Rozane Márcia Triches 
A relaÇão ENTRE O GRAU de INTEGRaÇÃo ECONÔMiCA E O SISTEMA DE SOLUÇÃo DE CONTROvÉRSIAS: UM ESTUdo COMPARATIVO ENTRE A UNIÃo EURopeia E O MERCOSUl.....................286

Luciane Klein Vieira e Elisa Arruda

THE RIGHTS TO MEMORY AND TRUTH IN THE INTER-AMERICAN PARADIGMS OF TRANSITIONAL JUSTICE: THE CASES OF BRAZIL AND CHILE 308

Bruno Galindo

Juliana Passos de Castro

A margem nacional de apreciação na Corte Interamericana de Direitos Humanos.325 Gilberto Schäfer, José Eduardo Aidikaitis Previdellie e Jesus Tupã Silveira Gomes

Novos Direitos FUNDAMENTAIS NO ÂMBITo DA UNASUL: ANÁLISE DAS AGENDAS DE Brasil E VENEZUELA À LUZ DO DIREITO À PAZ.

Pedro Pulzatto Peruzzo e Arthur Ciciliati Spada

A atuação do Grupo Mercado Comum frente À Criminalidade organizada transnacio-

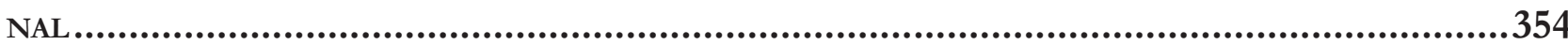

Sabrina Cunha Kesikowski, Luis Alexandre Carta Winter e Eduardo Biacchi Gomes

COUNTER-TERRORISM LEGISLATION AND TERRORIST ATTACKS: DOES HUMAN RIGHTS HAVE SPACE?

Heloisa Tenello Bretas e Daniel Damásio Borges

Territórios da ViolênCia de GÊNERo: NORMATIVA INTERNACIONAL E os Casos "CAMpo AlGodoeiro” (MÉXICO) - “Morro do Garrote” (BrasiL)

Eugênia Nogueira do Rêgo Monteiro Villa e Bruno Amaral Machado

O USO DE MECANISMOS INFORMAIS DE GOVERNANÇA GLOBAL E SUA APLICABILIDADE NAS LICITAÇÕES PÚBLICAS BRASILEIRAS.

Fabiano de Figueiredo Araujo e Paulo Afonso Cavichioli Carmona

Autonomia INSTITUCIONAL DA IGREJA CATÓliCA E A INGERÊNCIA INDEVIDA DO ESTADO BRASILEIRo POR EVENTUAIS Ilícitos CANÔNicos: ANÁlise do CASO de Formosa-GO, À luz do TraTADo Brasil-Santa SÉ de 2010 
A MORE TARGETED APPROACH TO FOREIGN DIRECT INVESTMENT: THE ESTABLISHMENT OF SCREENING SYSTEMS ON NATIONAL SECURITY GROUNDS

Carlos Esplugues Mota

IV. RESENHAS 467

Direito Internacional em Perspectiva Transcivilizacional de Yasuaki Onuma........469 Arthur Roberto Capella Giannattasio

Resenha do livro Space, Global Life: The Everyday Operation of International LaW and Development, de Luis Eslava .473

Matheus Gobbato Leichtweis

QUEM TEM MEDO DO PÓS-COLONIAL NO DIREITO INTERNACIONAL? UMA RESENHA DE "DECOLONISING INTERNATIONAL LAW: DEVELOPMENT, ECONOMIC GROWTH AND THE POLITICS OF UNIVERSAlity" de Sundhya Pahuja

Gabriel Antonio Silveira Mantelli

Direitos humanos COMO UM NOVO PROJETO PARA O Direito INTERNACIONAL? Notas sobre The Last Utopia, de Samuel Moyn .490 João Roriz 


\title{
Quem tem medo do pós-colonial no direito internacional? Uma resenha de "Decolonising international law: development, economic growth and the politics of universality" de Sundhya Pahuja*
}

\author{
Who's afraid of the postcolonial in \\ international law? A review of Sundhya \\ Pahuja's "Decolonising international law: \\ development, economic growth and the politics \\ of universality"
}

Gabriel Antonio Silveira Mantelli**

Em 2014, no Festival de Cinema de Berlim, um único filme trouxe para o solo europeu questões atinentes à realidade da África. Concerning Violence, ironicamente um documentário coproduzido por países do Primeiro Mundo, resgata o passado colonial ao apresentar os movimentos africanos de independência das décadas de 1960 e 1970 e acaba questionando, inclusive, as relações contemporâneas de poder entre os países do Norte e do Sul. O documentário, de extrema relevância, é construído em torno de trechos da obra de Frantz Fanon, importante intelectual que influenciou toda uma geração de pensadores/as pós-coloniais. Em sua obra seminal, os condenados da terra, ele afirma que a descolonização é um processo de violência e que "[n]a descolonização há, pois, a exigência de uma entrega completa da situação colonial”. ${ }^{1}$

No campo do direito internacional, há quem realize esse movimento de retorno ao colonial para desvendar certas estruturas que, ainda, vingam em nosso contexto global. Em sintonia com Fanon, temos Sundhya Pahuja que afirma que o movimento de descolonização pode ser encarado não somente do ponto de vista otimista do nacionalismo, como bradado nos movimentos de libertação política, mas, também com certo ceticismo. Nesse caso, a descolonização poderia ser encarada como uma nova forma de aprisionamento, agora jurídico, afinal, para se tornarem independentes e comporem a nova ordem mundial, as antigas colônias tiveram que adotar os modelos jurídicos estipulados pelas potências da época, normalmente suas antigas metrópoles. Assim, paradoxalmente, para resistirem à colonização, esses novos países acabaram por se entregar à epistemologia de seus antigos opressores.

No direito internacional, de fato, parecia que a temática da colonização havia sido deixada para trás em função de certa narrativa focada na vitória da globalização e dos direitos humanos. Todavia, uma agenda crítica tem trazido à tona o "outro lado da moeda" do direito internacional. Nessa Getulio Vargas (FGV Direito SP) com bolsa da CAPES. Graduado em Direito pela Universidade de São Paulo (USP). Integra o Núcleo de Direito Global e Desenvolvimento da FGV Direito SP. E-mail: gabrielmantelli@gmail.com

1 FANON, Frantz. Os condenados da terra. Lisboa: Ulisseia, 1961. p. 31.

2 Usa-se a expressão comumente utilizada pelos teóricos/as latino-americanos/as decoloniais, em que o lado escuro da modernidade seria a colonização. Neste sentido, ver: MI- 
agenda, o regime colonial e o imperialismo têm sido campos de estudo bastante fecundos para esses trabalhos, sobretudo por teóricos/as alinhados/as às Abordagens do Terceiro Mundo no Direito Internacional (Third World Approaches to International Law - TWAIL). ${ }^{3}$ Incluída nesse grupo (e particular a ele também), ${ }^{4} \mathrm{Pahu}-$ ja explora a temática do desenvolvimento no livro Decolonising internacional law: development, economic growth and the politics of universality. ${ }^{5}$

Publicado em 2011, pela Cambridge University Press, fruto do seu doutorado no Reino Unido e atuação como professora na Austrália, o livro é capaz, ainda hoje, de lançar importantes reflexões sobre o direito internacional no contexto dos países do Terceiro Mundo bem como tem papel relevante no contexto brasileiro de pesquisa em direito, colaborando para a construção de uma agenda crítica no direito internacional. O título do livro dá a tônica de um debate pós-colonial e a ênfase terceiro-mundista é perceptível no questionamento que abre o trabalho: "por que o direito internacional, da perspectiva do Terceiro Mundo, tem sido tão decepcionante?". De um lado, a noção de desapontamento que a autora procura explicitamente vincular ao direito internacional ilustra uma das marcas do livro: olhar o direito internacional para além do seu funcionamento normativo. Com isso, ela agrega ao estudo uma esfera contextual e interdisciplinar bastante reveladora de tensões e dinâmicas que o direito, isolado, talvez não fosse capaz de explicar. De outro lado, a escolha pelo termo Terceiro Mundo para designar o que hoje se define, na

GNOLO, Walter. The darker side of western modernity: global futures, decolonial options. Durham: Duke University Press, 2011.

3 Ver genericamente: MUTUA, Makau. What is TWAIL? American Society of International Law, Proceeding of the 94th Annual Meeting, p. 31-39, 2000; CHIMNI, Bhupinder S. Third world approaches to international law: a manifesto. International Community Law Review, Leiden, v. 8, p. 3-27, 2006; GALINDO, George Rodrigo Bandeira. A volta do terceiro mundo ao direito internacional. Boletim da Sociedade Brasileira de Direito Internacional, Belo Horizonte, v. 1, n. 119-124, p. 46-68, 2013; ANGHIE, Antony. Imperialism and international legal theory. In: ORFORD, Anne; HOFFMAN, Florian (Org.). The Oxford Handbook of the Theory of International Law. Oxford: Oxford University Press, 2015. p. 156-173.

4 Ela se posiciona de forma singular tanto ao pensamento mainstream quanto ao próprio terceiro-mundista. Acadêmicos/as das principais correntes celebram o fim da $2^{\text {a }}$ Guerra Mundial como o fim do imperialismo, ao passo que os terceiro-mundistas criticam suas continuidades. Para Pahuja, o direito internacional do pós- $2^{a}$ Guerra Mundial é tanto imperialista quanto emancipador.

5 PAHUJA, Sundhya. Decolonising international law: development, economic growth and the politics of universality. Cambridge: Cambridge University Press, 2011. academia e fora dela, como países em desenvolvimento ou Sul Global. A própria autora dedica um anexo do livro para explicar essa escolha, justificando que a intenção é remontar ao movimento anticolonial e demarcar uma dimensão política que outras nomenclaturas, segundo ela, não são capazes de atrelar. Ao fazê-lo, Pahuja deixa, ainda mais evidente, o lugar de onde o trabalho foi elaborado.

É interessante atentar para o debate do pós-colonialismo refletido na obra. A perspectiva pós-colonial foi "[i]niciada por aqueles autores qualificados como intelectuais da diáspora negra ou migratória” e, após esse primeiro momento, "expande-se geograficamente [...], fazendo dos trabalhos de autores como Homi Bhabha, Edward Said, Gayatri Chakravorty Spivak ou Stuart Hall e Paul Gilroy referências recorrentes". ${ }^{6}$ De um lado, o pós-colonialismo emerge "tanto [como] um ponto de encontro quanto um campo de batalha entre uma variedade de disciplinas e teorias"; 7 de outro, "contém uma crítica, implícita ou explícita, aos silêncios das análises pós-coloniais na primeira acepção". ${ }^{8}$ No trabalho de $\mathrm{Pa}$ huja, cujo esforço interdisciplinar é louvável, esse viés é bastante demarcado, tanto pela temática da descolonização quanto pelo tom crítico da autores, pensadores/as pós-coloniais como Dipesh Chakrabarty, Partha Chatterjee e Gayatri Spivak são utilizados/as como referenciais teóricos.

Se, de um lado, podemos observar o contexto do trabalho, que aponta para uma obra crítica de direito internacional, influenciada pelo pós-colonialismo; de outro, podemos demarcar a estrutura de Decolonising international law. Ele é dividido em seis capítulos, com dois apêndices, um que já apresentei, e outro com o discurso de Harry Truman que abre o debate global sobre desenvolvimento. Os dois primeiros capítulos introduzem a matéria a ser trabalhada no livro, basicamente a concepção do desenvolvimento enquanto um discurso que legitima uma nova racionalidade no direito internacional. Para essa tarefa, os três capítulos seguintes apresentam três casos relevadores dessa nova dinâmica, o que abordo, oportunamente, e apresenta um capítulo

6 COSTA, Sérgio. Desprovincializando a sociologia: a contribuição pós-colonial. Revista Brasileira de Ciências Sociais, São Paulo, v. 21, n. 60, p. 117, fev. 2006.

7 GANDHI, Leela. Postcolonial theory: a critical introduction. Nova York: Columbia University Press, 1988. p. 3.

8 SANTOS, Boaventura de Sousa. Entre Próspero e Caliban: colonialismo, pós-colonialismo e interidentidade. Novos Estudos CEBRAP, São Paulo, n. 66, p. 26, jul. 2003. 
para a conclusão. No livro, temos como marco o fim da Segunda Guerra Mundial e o estabelecimento de uma nova ordem global, em que o direito internacional passou a ser bastante influenciado pela hegemonia dos Estados Unidos da América e pelo discurso universal de desenvolvimento. Segundo ela, a partir desse momento, o direito internacional teria passado a constituir um complexo ideológico-institucional que, ao mesmo tempo, seria capaz de abarcar tanto o imperialismo quanto a resistência a ele. E que, desde então, o direito internacional haveria sofrido tanto uma ruptura quanto um continuísmo das dinâmicas do colonialismo: teria existido o nascimento de novas estruturas institucionais que deram continuidade a velhas práticas imperialistas. A principal tese da obra, por sua vez, gira em torno da ideia de que a descolonização, o desenvolvimento moderno e a universalização do direito internacional teriam produzido uma nova forma de poder ou, nas palavras de Pahuja, uma nova racionalidade (ruling rationality, no original). Essa nova racionalidade teria capturado as reivindicações do Terceiro Mundo e, nesse processo, teria minado o potencial radical deles. O principal efeito desse cenário seria, então, a emergência de um quadro regulatório, universalmente aplicado, que tem subsumido a promessa criativa do direito internacional.

Para desenvolver essa tese, Pahuja se utiliza de três casos relevadores: primeiro, o movimento de descolonização que levou à formação do Estado-nação desenvolvimentista; segundo, a transformação da soberania permanente sobre recursos naturais em regras de proteção a investidores estrangeiros, elevando-os à categoria de sujeito do direito internacional; e, terceiro, a transformação do império do direito internacional em internacionalização do império do direito (rule of law) enquanto uma estratégia do desenvolvimento. Por meio dessas três esferas críticas - a nação, os recursos naturais e a economia política — o que se argumenta é que a antiga missão civilizadora do colonialismo, então sob o duplo mandato de explorar recursos para o benefício mútuo da colônia e da metrópole, acabou, também, persistindo sob o pretexto da construção de uma nação desenvolvida e da integração internacional. "Descolonizar" o direito internacional, nesse contexto, teria como propósito demarcar a dualidade desse direito — suas tendências imperialistas e emancipatórias.

Tal dualidade produz, no âmago do direito internacional, o que a autora chama de instabilidade crítica. De um lado, o direito internacional seria, em si, pós- -colonial. Quer dizer, assim, que o direito internacional não precederia as categorias em que ele se funda (como as noções de Estado e universalidade, por exemplo). Ao contrário, o direito internacional produziria e modelaria essas categorias; seria um gerador e mantenedor de um conteúdo particular para o universal. Então, na medida em que o direito internacional não poderia ser considerado universal, mas sim como detentor de valores, categorias e formas sociais particulares ou ocidentais, teríamos uma instabilidade crítica. Com esse raciocínio, o que Pahuja procura desvendar é a maneira como certos atores e determinadas narrativas acabam silenciados. Isto porque, segundo ela, essa instabilidade cria categorias que tornam invisíveis aqueles que não se conformam com as particularidades do direito internacional. De outro lado, essa instabilidade, e aqui retomamos o questionamento inicial da autora, advém da lacuna existente entre a promessa de justiça do direito internacional e a personificação do direito internacional em uma agenda regulatória fechada. Lacuna esta que ela denomina de política do direito internacional.

As ideias apresentadas por Pahuja são bastante provocadoras, tanto para pensadores/as tradicionais do direito internacional quanto, inclusive, para ativistas de direitos humanos que se utilizam da narrativa do direito ao desenvolvimento. O título que desta resenha, também com esse viés provocativo, se inspira na peça teatral Who's afraid of Virginia Woolf?, obra de Edward Albee que tem despertado reflexões sobre o caráter dúbio das dinâmicas humanas e o falso otimismo que criamos em torno da sociedade moderna. O desenvolvimento, que, por seu caráter transcendental, pode ser encarado tanto uma nova "religião", como Pahuja aponta ao retomar a obra de Gilbert Rist, quanto um discurso, se referindo à obra de Arturo Escobar, tem, de fato, subsumido a promessa do direito internacional pela inclusão e justiça genuínas — basta um olhar aguçado para o contexto que nos rodeia.

O pós-colonialismo, por sua vez, traz consigo um potencial de resistência que tenciona o próprio direito internacional. Com o trabalho de Pahuja, ao tomarmos consciência dessa instabilidade crítica do direito internacional, temos um interessante ferramental que pode auxiliar a elaboração de categorias jurídicas anti-imperialistas e emancipatórias. No caso de produções situadas desde a América Latina, o potencial de ganho analítico é maior e ainda pouco explorado no direito internacional pensado a partir do Brasil. No caso do 
pensamento decolonial, ${ }^{9}$ essa nova literatura, elaborada no seio das ciências sociais, também tem questionado o passado colonial, dessa vez de forma mais aprofundada e sintonizada com ideários emancipatórios de resistência. Encarar esses dilemas históricos, no caso de trabalhos interessados em compreender o direito internacional na América Latina, é tarefa da ordem do dia.

De todo modo, temos em mãos uma importante contribuição para nosso campo de estudo. Bastante oportuno e metodologicamente interessante, Decolonising international law deveria, por fim, constar nas sugestões de leitura dos cursos de graduação e pós-graduação de direito, especialmente para aqueles programas que se interessem em debater narrativas alternativas no direito internacional.

\section{RefERÊNCIAS}

ANGHIE, Antony. Imperialism and international legal theory. In: ORFORD, Anne; HOFFMAN, Florian (Org.). The oxford handbook of the theory of international law. Oxford: Oxford University Press, 2015.

CASTRO-GÓMEZ, Santiago; GROSFOGUEL, Ramón (Ed.). El giro decolonial: reflexiones para una diversidad epistémica más allá del capitalismo global. Bogotá: Siglo del Hombre Editores, 2007.

CHIMNI, Bhupinder S. Third world approaches to international law: a manifesto. International Community Law Review, Leiden, v. 8, p. 3-27, 2006.

COSTA, Sérgio. Desprovincializando a sociologia: a contribuição pós-colonial. Revista Brasileira de Ciências Sociais, São Paulo, v. 21, n. 60, p. 117-134, fev. 2006.

FANON, Frantz. Os condenados da terra. Lisboa: Ulisseia, 1961.

GALINDO, George Rodrigo Bandeira. A volta do terceiro mundo ao direito internacional. Boletim da Sociedade Brasileira de Direito Internacional, Belo Horizonte, v. 1, n. 119-124, p. 46-68, 2013.

GANDHI, Leela. Postcolonial theory: a critical introduction. Nova York: Columbia University Press, 1988.

9 Ver genericamente: CASTRO-GÓMEZ, Santiago; GROSFOGUEL, Ramón (Ed.). El giro decolonial: reflexiones para una diversidad epistémica más allá del capitalismo global. Bogotá: Siglo del Hombre Editores, 2007.
MIGNOLO, Walter. The darker side of western modernity: global futures, decolonial options. Durham: Duke University Press, 2011.

MUTUA, Makau. What is TWAIL?. American Society of International Law, Proceeding of the 94th Annual Meeting, p. 31-39, 2000.

PAHUJA, Sundhya. Decolonising international law: development, economic growth and the politics of universality. Cambridge: Cambridge University Press, 2011.

SANTOS, Boaventura de Sousa. Entre Próspero e Caliban: colonialismo, pós-colonialismo e interidentidade. Novos Estudos CEBRAP, São Paulo, n. 66, p. 23-52, jul. 2003. 
Para publicar na Revista de Direito Internacional, acesse o endereço eletrônico www.rdi.uniceub.br ou www.brazilianjournal.org.

Observe as normas de publicação, para facilitar e agilizar o trabalho de edição. 